\title{
Diseño de un instrumento para valorar la comprensión alcanzada en divisibilidad por futuros profesores de matemática
}

\section{Desenho de um instrumento para valorar a compreensão que apresentam futuros professores de matemática acerca da divisibilidade}

\author{
Ricardo Fabián Espinoza* \\ ORCID iD 0000-0001-8921-6956 \\ Marcel David Pochulu** \\ ORCID iD 0000-0003-2292-4178
}

\begin{abstract}
Resumen
La investigación que se presenta en este artículo, tuvo por objetivo valorar la comprensión que han alcanzado futuros profesores de matemática sobre objetos matemáticos de la divisibilidad. Para tal fin, se diseñó y validó un instrumento de indagación, el que se constituye en un insumo valioso para revisar no sólo las prácticas que llevan a cabo los futuros profesores de matemática, sino también, para explorar y potenciar la faceta del conocimiento epistémico sobre la divisibilidad en los formadores de profesores. La resolución de cada situación problema que compone el instrumento deja al descubierto funciones semióticas actuativas y argumentativas, y una amplia red de relaciones conceptuales involucradas entre objetos matemáticos primarios, lo que hace posible valorar la comprensión lograda de la divisibilidad.
\end{abstract}

Palabras clave: Enseñanza de la divisibilidad. Tipos de problemas sobre divisibilidad. Análisis ontológico y semiótico. Comprensión de la divisibilidad. Formación de profesores.

\section{Resumo}

Este artigo apresenta uma pesquisa que teve como objetivo valorar a compreensão que apresentam futuros professores de matemática sobre os objetos matemáticos relacionados à divisibilidade. Para tanto, foi elaborado e validado um instrumento de investigação, que constitui um valioso contributo para revisar não apenas as práticas realizadas pelos futuros professores de matemática, mas, também, para explorar e aprimorar a faceta do conhecimento epistêmico sobre divisibilidade em formadores de professores.

A resolução de cada situação-problema que compõe o instrumento expõe as funções semióticas performativa e argumentativa, e uma grande rede de relações conceituais envolvidas entre objetos matemáticos principais, tornando-se possível avaliar a compreensão adquirida pelos professores sobre a divisibilidade.

Palavras-chave: Ensino de divisibilidade. Tipos de problemas sobre divisibilidade. Análise semiótica e ontológica. Compreensão da divisibilidade. Formação de professores.

* Dr. en Ciencias Humanas y Sociales, UNaM, Argentina. Docente-Investigador de la UNNE, Corrientes, Provincia de Corrientes, República Argentina. Dirección postal: 25 de mayo 1995, Departamento 11, Corrientes, República Argentina, código postal 3400. E-mail: rrfespinoza@gmail.com.

** Dr. en Didáctica de la Matemática, UNED, España. Docente-Investigador de la UNVM, Villa María, Provincia de Córdoba, República Argentina. Dirección postal: Río Tunuyán 42, Barrio Vista Verde, Villa María, Provincia de Córdoba, República Argentina, código postal 5900. E-mail: marcelpochulu@gmail.com. 


\section{Introducción}

A partir del siglo XX, la Teoría de Números ocupó un amplio espectro en la matemática, destacándose el estudio de la Divisibilidad en el conjunto de los Números Naturales. En la educación formal, el estudio de esta temática se inicia en la escuela primaria o básica, cuando los alumnos trabajan con tareas que involucran divisores, múltiplos, factores, criterios de divisibilidad, factorización, máximo común divisor, mínimo común múltiplo, clasificación de números en primos o compuestos, entre otros.

En el siguiente nivel educativo (educación secundaria, nivel medio o bachillerato, dependiendo la denominación que le otorgan los diferentes países) se profundizan conceptos, definiciones, algoritmos y rutinas con renovados problemas sobre Divisibilidad en el conjunto de los Números Naturales, y se extienden algunas propiedades al conjunto de los Números Enteros.

Al respecto, diversos reportes de investigación (DUBINSKY, 1991; VERGNAUD, 1994; ZAZKIS; GADOWSKY, 2001; BODÍ, 2006; BODÍ; VALLS; LLINARES, 2007; ESPINOZA, 2012; VALLEJOS-VARGAS, 2012), muestran que los alumnos, en esta etapa, continúan teniendo dificultades para resolver tareas sencillas vinculadas con este contenido matemático. Los reportes expresan, por ejemplo, que los estudiantes frecuentemente asocian el concepto de divisor con la operación de dividir y el concepto de múltiplo con la operación de multiplicar, lo cual muestra una comprensión incompleta del concepto de factor, entre otros objetos matemáticos involucrados. Otros, en cambio, realizan un intercambio constante e incoherente entre el lenguaje formal y no formal, como por ejemplo, la expresión ser divisible (relación entre dos números) es sustituida por ser dividido (un número que puede ser dividido por otro).

Culminando la educación media, secundaria o el bachillerato, algunos estudiantes escogerán carreras profesionales para ser maestros o profesores de matemática. Nuevamente serán abordados, con mayor rigurosidad, los contenidos de la Teoría de Números en las primeras materias, cátedras o asignaturas de la formación de profesores. El National Council of Teachers of Mathematics (NCTM, 1989) explica que el abordaje de esta temática proporciona una comprensión profunda a los maestros de las propiedades y las estructuras numéricas. Asimismo, el informe de la Conference Board of the Mathematical Sciences (CBMS, 2001) insiste sobre esta recomendación, pero referida a la formación de profesores de matemática para la escuela secundaria.

Las investigaciones relacionadas con la Divisibilidad y futuros maestros o profesores 
de matemática, en el nivel superior o universitario, vuelven a ser numerosas y con resultados dispares. Por ejemplo, Zazkis (2001) explora la red de relaciones que establecen diecinueve estudiantes de magisterio entre los términos de múltiplo, divisor y factor, en el ámbito de un curso de formación, en el que se abordaron temas como el algoritmo de la división, divisores, factores, múltiplos, reglas de divisibilidad, números primos, números compuestos, factorización en números primos, mínimo común múltiplo y máximo común divisor. Los resultados sugieren que las relaciones, que matemáticamente parecen claras y sencillas, representan una red compleja para los estudiantes. Al resolver los problemas, las aproximaciones que tienen los alumnos también demuestran que no utilizan totalmente las vinculaciones matemáticas de estos conceptos, dado que la mayoría de las veces son débiles o incompletas.

En contraposición, se tiene la tesis doctoral desarrollada por Feldman (2012), quien describe el proceso llevado a cabo por 59 maestros en formación en la comprensión de tópicos de Teoría Elemental de Números (factores, divisibilidad, máximo común divisor y mínimo común múltiplo). Al analizar los resultados, la investigadora señala que el estudio realizado proporciona evidencias de que los maestros en formación pueden desarrollar una comprensión profunda y relacional de este tópico. Sugiere que, en investigaciones futuras sobre comprensión de objetos matemáticos de la Teoría de Números, se preste atención al trabajo que los maestros desarrollan durante los períodos de instrucción en las aulas. Asimismo, señala que las tareas matemáticas que requieren de la descomposición factorial prima de un número, como herramienta fundamental de la Teoría de Números, deben ser investigadas por los efectos que producen en la comprensión de los maestros en formación.

En consecuencia, el objetivo de este trabajo fue diseñar y validar un instrumento que permita valorar la comprensión que han alcanzado los futuros maestros y profesores de matemática, cuando inician estudios superiores o universitarios sobre la Divisibilidad. Se pretende, a su vez, que el instrumento sea útil para realizar un diagnóstico de las relaciones matemáticas construidas, o no, entre los tópicos centrales que conforman la Teoría Elemental de Números. Con este diagnóstico inicial, el profesor formador podrá diseñar tareas partiendo de lo que, efectivamente, comprendieron los futuros maestros y profesores, logrando un proceso cognitivo más relevante y una comprensión más profunda sobre la Divisibilidad.

\section{Marco teórico}

La noción de comprensión tiene múltiples acepciones y numerosos investigadores en 
Educación Matemática la caracterizan (GODINO, 2000, 2003; FONT, 2011; PINO-FAN; GODINO; FONT, 2011, entre otros). En este trabajo se la entiende del siguiente modo, a partir de una adaptación expresada en el INFD (2010, p. 122):

\begin{abstract}
Comprender un objeto matemático significa (...) producir, organizar y reorganizar la red de relaciones que se deben establecer en la resolución de una situación problemática (intra y extra-matemática) que "obliga" al funcionamiento del objeto, los procedimientos o técnicas que se despliegan para resolverla, las definiciones, propiedades, argumentos que validan las acciones realizadas, todas ellas soportadas y reguladas por el lenguaje (...), propio de la Matemática, y la lengua natural.
\end{abstract}

Esta concepción involucra al profesor en el diseño de buenas tareas de matemática para la comprensión y una gestión de la clase apropiada, para que el estudiante pueda ser capaz de articular coherentemente y establecer relaciones entre seis elementos: las situaciones problemas en las que participa el objeto matemático, los conceptos, las propiedades, los procedimientos, los argumentos y el lenguaje. No obstante, tal como se señala en INFD (2010), al reflexionar sobre esta acepción, la pregunta que subyace de fondo es: ¿cómo sabrán los profesores y los estudiantes que se ha alcanzado la comprensión de determinado objeto matemático?, ¿cómo se recaba información sobre la comprensión alcanzada por un estudiante?

Para encontrar respuestas a estas preguntas se recurre a constructos y herramientas del Enfoque Ontológico y Semiótico del conocimiento e instrucción matemática (EOS) que proponen Godino (2000, 2003) y Godino, Batanero y Font (2007). El EOS considera que toda práctica o actividad matemática está centrada en la resolución de problemas (en el sentido más amplio de su acepción, los cuales van desde simples ejercicios a instancias de modelación), y se pueden encontrar algunos o todos de los siguientes elementos primarios: situaciones problemas, conceptos, propiedades o proposiciones, procedimientos, argumentaciones y lenguaje.

Estos seis objetos primarios que están presentes en una práctica matemática se relacionan entre sí formando configuraciones epistémicas o cognitivas (Figura 1). Las mismas son entendidas como las redes de objetos intervinientes y emergentes de los sistemas de prácticas, y las relaciones que se establecen entre los mismos, y constituyen los elementos del significado de un objeto matemático particular.

Las configuraciones pueden ser epistémicas o instruccionales, si son redes de objetos institucionales (extraídas de un texto escolar, obtenidas de la clase que imparte un profesor etc.); o cognitivas, si representan redes de objetos personales (actividad de los estudiantes). Tanto los sistemas de prácticas como las configuraciones se proponen como herramientas teóricas para describir los conocimientos matemáticos, en su doble versión, personal e 
institucional (GODINO; BATANERO; FONT, 2007).

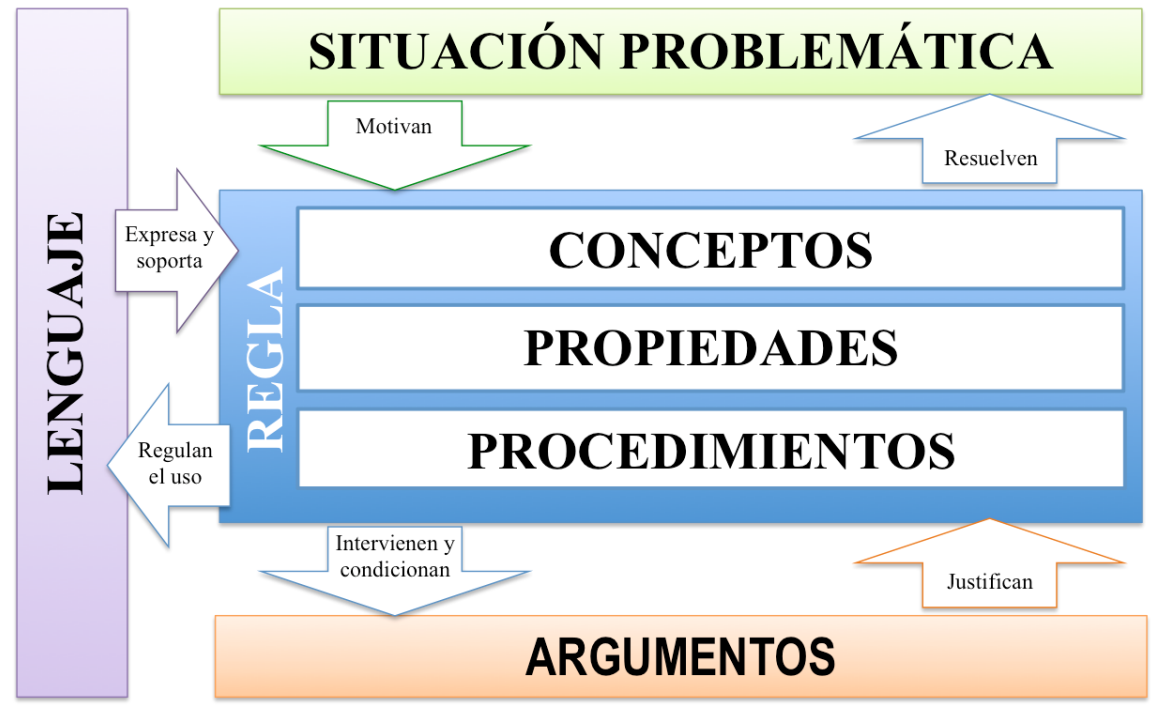

Figura 1 - Configuración Epistémica

Fuente: Adaptación de D’Amore, Font y Godino (2007, p. 59)

Se puede advertir que, en las configuraciones epistémicas, las situaciones problemáticas son las que le dan origen a la propia actividad matemática y las que vienen a motivar el conjunto de reglas que aparecen en ella. El lenguaje, por su parte, sirve de instrumento para accionar en la actividad matemática que acontece. Los argumentos, en tanto, se entienden como prácticas que aparecen para justificar las definiciones, procedimientos y proposiciones, las que están reguladas por el uso del lenguaje que, por su parte, sirve de instrumento para la comunicación. Cada objeto matemático, dependiendo del nivel de análisis que se quiera hacer, puede estar compuesto por entidades de los restantes tipos. Un argumento, por ejemplo, puede poner en juego conceptos, proposiciones, procedimientos o combinaciones entre ellos y, obviamente, está soportado por el lenguaje.

La finalidad de estas configuraciones es analizar las prácticas matemáticas describiendo su complejidad ontosemiótica. Esto induce a detectar y explicar conflictos semióticos que se pueden producir cuando se lleva a cabo estas prácticas en un determinado proceso de estudio.

En una situación ideal y en una institución dada, Godino (2003) sostiene que un sujeto comprende el significado de un objeto o se apropia del significado de un concepto, si es capaz de reconocer los problemas, definiciones, procedimientos, argumentaciones, propiedades y representaciones características, relacionarlo con los restantes objetos matemáticos en toda la variedad de situaciones planteadas por la institución correspondiente.

Los distintos objetos primarios no resultan aislados entre sí, sino que se vinculan a 
través de las funciones semióticas construidas entre ellos. D’Amore, Font y Godino (2007) indican que una función semiótica está caracterizada por una correspondencia entre un antecedente (expresión, significante o representante) y un consecuente (contenido, significado, representado) que establece un sujeto, persona o institución de acuerdo con cierto criterio. La correspondencia (representacional o instrumental) se constituye entre dos objetos (ostensivos o no-ostensivos), cuando uno de ellos se pone en lugar del otro o bien uno es usado por otro. Tanto el objeto inicial como final, en una función semiótica, pueden estar constituidos por uno o varios de los objetos primarios, los que pueden desempeñar el papel de expresión o de contenido en las funciones semióticas (GODINO, 2003).

Con la noción de función semiótica, se evidencia el carácter netamente relacional de la actividad matemática y de los procesos que difunden el conocimiento matemático. Como podrá observarse en la sección de los resultados, de este trabajo emerge una clasificación de funciones semióticas establecidas por futuros profesores: actuativas y argumentativas (conceptuales, proposicionales, con contraejemplos y deductivas).

Font (2002) manifiesta que el hecho de comprender un objeto (ostensivo o no, concreto o abstracto) por parte de un sujeto (persona o institución) se interpreta en términos de las funciones semióticas que tal sujeto puede establecer. Explica que cada función semiótica implica un acto de semiosis por parte de un agente interpretante y forma un conocimiento; razón por la cual, las funciones semióticas poseen un gran potencial como herramientas metodológicas al momento de estudiar significados, particularmente, los significados personales construidos por los estudiantes.

\section{Metodología}

La metodología de investigación de este trabajo está basada en el diseño (COBB; GRAVEMEIJER, 2008) y fundamentada en el empleo de herramientas del EOS (GODINO, 2000, 2003, 2013; GODINO; BATANERO; FONT, 2007). En particular, se consideraron las nociones de configuración epistémica (para modelizar los sistemas de prácticas institucionales de referencia), configuración cognitiva (para modelizar los sistemas de prácticas personales de los estudiantes) y función semiótica (para establecer relaciones conceptuales en sistemas de prácticas institucionales y personales).

En las investigaciones basadas en el diseño se deben considerar tres etapas: (1) planificación del experimento, (2) experimentación y (3) análisis retrospectivo de los datos generados en el experimento (COBB; GRAVEMEIJER, 2008). En este estudio, las etapas se 
repitieron cíclicamente para poder realizar los ajustes necesarios al diseño del instrumento.

Para la primera etapa (planificación del experimento), se elaboró un marco epistémico y didáctico de referencia, modelizado a partir de configuraciones epistémicas referidas a la Divisibilidad. No se trató de un listado de contenidos desvinculados, como habitualmente puede observarse en textos escolares, sino de un conjunto de objetos matemáticos primarios y las relaciones establecidas entre ellos. En particular, se tuvieron en cuenta:

- Los trabajos que están centrados en el estudio didáctico-matemático de redes de relaciones entre objetos matemáticos básicos de la Teoría de Números (ETCHEGARAY, 1998; ESPINOZA, 2012).

- Las investigaciones que se han llevado a cabo con maestros o profesores durante la formación inicial (ZAZKIS, 2001; BODÍ, 2006; LÓPEZ, 2016) sobre Divisibilidad.

- Los informes que se han realizado en las escuelas, con estudiantes o docentes (maestros o profesores) en ejercicio de la profesión (BODÍ; VALLS; LLINARES, 2007; VALLEJOS-VARGAS, 2012) sobre Divisibilidad.

- Los reportes que ponen énfasis en la importancia de las distintas formas de representación de los números en la comprensión de los contenidos de la Divisibilidad (ZAZKISY; GADOWSKY, 2001; BROWN, 2002; ZAZKIS; LILJEDAHL, 2004; BODÍ, 2006; BODÍ; VALLS; LLINARES, 2007).

- Las investigaciones que están relacionadas con la comprensión de un objeto matemático (DISTÉFANO, 2017; PINO-FAN; GODINO; FONT, 2013, entre otras).

- Los libros de texto escolares que se emplean para la enseñanza de la Divisibilidad en Argentina (ocho destinados a estudiantes de escuela secundaria, básica o media y cinco para la formación de maestros y profesores de matemática).

- Recomendaciones que devienen de los diseños y lineamientos curriculares jurisdiccionales de Argentina sobre Divisibilidad.

A partir de las configuraciones epistémicas anteriores, se diseñó el primer instrumento destinado a recuperar información sobre la comprensión alcanzada por los futuros maestros y profesores de matemática, cuando ingresan al nivel superior o universitario. Junto al instrumento se construyó una matriz, o rúbrica, que contiene las prácticas matemáticas institucionales determinadas a priori para cada situación problemática, según diferentes desempeños: novato, aprendiz, experto y distinguido.

Para diferenciarlos, se tuvieron en cuenta los siguientes aspectos: la pertinencia, economía o adecuación de los objetos de la Divisibilidad empleados en la resolución (especialmente procedimientos); la utilización de conocimientos adecuados como 
definiciones, proposiciones y propiedades en la resolución de los problemas; y la incorporación de argumentos apropiados para validar las distintas producciones.

Arends (2004) sostiene que a través de las rúbricas se logra una descripción detallada del tipo de desempeño esperado por parte de los estudiantes. Por tal razón, la rúbrica se utilizó como complemento del análisis ontosemiótico del instrumento de indagación.

Posteriormente, se realizó una prueba piloto (etapa de experimentación) con estudiantes del primer año del Profesorado en Matemática del Instituto Superior de Formación Docente (ISFD) Dr. Juan Pujol, de la ciudad de Corrientes, Argentina. El propósito de esta prueba llevó a reelaborar las consignas que podrían estar produciendo conflictos semióticos o dificultades de comprensión de términos o palabras, ajustar tiempo de resolución y las prácticas matemáticas que forman parte de la matriz de desempeño (etapa de análisis retrospectivo de los datos).

Culminada la fase anterior, se inició un segundo ciclo de diseño. Para ello, se ajustó el instrumento de indagación y se realizó una validación de su contenido usando valoración experta de pares académicos (cuatro docentes e investigadores idóneos en el área de Matemática y Educación Matemática, con el grado de doctor). En la etapa de experimentación se realizó una exploración con futuros profesores de matemática que iniciaban sus estudios en la Universidad Nacional del Nordeste (UNNE), de la ciudad de Corrientes, Argentina.

El tercer y último ciclo de diseño del instrumento implicó ajustes que devienen del análisis didáctico de los sistemas de prácticas, desarrollados por futuros maestros y profesores al enfrentarse con la resolución de las situaciones problemáticas, y atendiendo a las observaciones y recomendaciones realizadas por los pares académicos. La focalización en la identificación de relaciones conceptuales, en las prácticas personales, posibilitó achicar el número de problemas del instrumento, eligiendo un representante entre aquellos que promovían el mismo tipo de dichas relaciones.

En la etapa de planificación de este tercer ciclo de diseño, se puntualizaron las principales relaciones entre objetos matemáticos que quedan al descubierto en las configuraciones cognitivas, definidas en términos de funciones semióticas. Además, se mejoraron sustancialmente las prácticas matemáticas institucionales de la matriz de desempeño, merced al análisis de las producciones de los alumnos que trabajaron con la segunda versión del instrumento.

La etapa de experimentación se llevó a cabo a principios del año 2018, en una de las primeras clases de Álgebra, con veinte futuros profesores de matemática de la UNNE, durante dos horas de trabajo. Luego de haber analizado las prácticas personales y confeccionar las 
configuraciones cognitivas, se convocó a varios de los futuros profesores para pedirles la ampliación de algunas producciones, relacionadas con la falta de claridad en el uso del lenguaje, la aplicación de ciertos procedimientos o algoritmos, el empleo de definiciones, propiedades o deducciones, la ausencia de fundamentación en las respuestas, entre otras.

Finalizado el tercer ciclo de diseño, se analizaron las configuraciones cognitivas de los futuros profesores de matemática, detectando las funciones semióticas que establecen en la resolución de las situaciones problemáticas del instrumento. Asimismo, se determinaron redes de relaciones conceptuales involucradas en esas funciones semióticas. Teniendo en cuenta la variedad y complejidad de los objetos matemáticos implicados en las funciones semióticas y de la red de relaciones establecidas entre los mismos, se realizó una clasificación de las mismas, lo que permitió valorar la comprensión que alcanzaron los profesores destinatarios en Divisibilidad.

\section{Resultados}

Del marco epistémico y didáctico de referencia se recuperan los siguientes tipos de situaciones problemáticas sobre Divisibilidad, expuestas en Espinoza (2019), y que están presentes en los libros de texto y reportes de investigación sobre este objeto matemático:

- Dado el cardinal de una colección que se pretende subdividir en subcolecciones equipotentes, determinar el número de subcolecciones de la partición, el cardinal de cada subcolección y el resto.

- Determinar si un número $a$ es divisor o factor de otro $b$, cuando $b$ está expresado en base decimal, como producto de factores primos, con base en el algoritmo de la división o la propiedad distributiva.

- Determinar si a, número natural o entero, está expresado como producto de factores primos, con base en el algoritmo de la división o en la propiedad distributiva.

- Determinar si 0 es divisor o múltiplo de todos los números.

- Determinar si 1 es divisor o múltiplo de todos los números.

- Caracterizar las fracciones $\frac{a}{b}$ y $\frac{b}{a}$, cuando a es divisor de $\mathrm{b}$, siendo a y $\mathrm{b}$ números no simultáneamente nulos.

- Determinar en qué condiciones, en una división, el divisor de la división es divisor del dividendo. 
- Hallar múltiplos de un número.

- Hallar un número conociendo una lista finita, exhaustiva y ordenada de sus múltiplos.

- Hallar todos los divisores de un número.

- Dados los divisores (naturales o enteros), encontrar el número correspondiente.

- Decidir si un número es primo, compuesto, cuadrado perfecto.

- Determinar la cantidad de divisores de un número.

- Determinar la paridad de la cantidad de divisores de un número.

- Hallar un número con una determinada cantidad de divisores.

- Encontrar el mínimo común múltiplo de dos o más números.

- Encontrar el máximo común divisor de dos o más números.

- Determinar si dos números son coprimos.

Las situaciones problemáticas que componen el instrumento de indagación son, en general, problemas particulares de algún tipo de tareas de Divisibilidad involucrados en el marco epistémico de referencia. Fueron adaptadas de las que se proponen en diferentes investigaciones (ZAZKIS; CAMPBELL, 1996; ZAZKIS, 2000, 2001; ZAZKIS; GADOWSKY, 2001; BROWN, 2002; ZAZKIS; LILJEDAH, 2004; BODÍ, 2006; BODÍ; VALLS; LLINARES, 2007; LÓPEZ, 2016) y las que están presentes en los libros de texto escolares que abordan la Divisibilidad.

Estas adaptaciones implicaron modificaciones en el enunciado de las consignas para que la resolución admitiera diferentes posibilidades de exploración y argumentación. En consecuencia, permitiría a los futuros maestros o profesores de matemática tomar decisiones, organizar sus intentos o modos para abordar la resolución, recurrir a heurísticas o utilizar distintas habilidades generales matemáticas, reflexionar sobre sus intentos para sostenerlos o descartarlos, establecer una manera de explicar el por qué de la respuesta y validar las conjeturas que emergen del proceso. Este proceso se asimila al trabajo del matemático, lo que legitima el tipo de actividad que se espera realizaría el profesor en el aula, y pone en evidencia las relaciones entre objetos primarios que son necesarias para valorar la comprensión alcanzada por los sujetos.

Asimismo, se consideró que el instrumento de indagación no tuviera demasiada cantidad de tareas o que demandara mucho tiempo para su desarrollo, pues provocaría que los futuros profesores dejaran inconclusas las resoluciones. En total se incluyeron diez situaciones problemáticas, las que son enunciadas a continuación:

Problema 1: a) ¿3 es divisor de 30?, ¿y de 473?, b) ¿3 es factor de 30?, c) ¿441 es 
múltiplo de 7 ? Fundamenta tu respuesta.

Problema 2: Si se divide al número $a$ por el número $b$, ¿existe alguna condición para que $b$ sea divisor de $a$ ? Justifica tu respuesta.

Problema 3: Todos los múltiplos de un número, comprendidos entre 370 y 460 son: 380, 399, 418, 437 y 456. ¿De qué número se trata?, ¿es único? Explica cómo lo/s encontraste y fundamenta tu respuesta.

Problema 4: 15a45 es un número de 5 cifras, ¿existe algún valor de $a$ para que este número sea divisible por 3 ? En caso de que tu respuesta fuera afirmativa, indica todos los posibles valores de a. Explica cómo lo hiciste y justifica tu respuesta.

Problema 5: se sabe que, si $a$ y $b$ son números naturales y $a$ es divisor de $b$, siempre a es menor o igual que $b$. ¿Sucede lo mismo si los números fueran enteros? Identifica todas las posibilidades para este caso y justifica tu respuesta.

Problema 6: ¿es posible que dos números distintos tengan los mismos divisores? Si tu respuesta es afirmativa, indica en qué condiciones eso ocurre. Justifica tu respuesta.

Problema 7: teniendo en cuenta que: $187=11 \times 17$, ¿son correctas las siguientes afirmaciones?: a) 17 es divisor de $11 \times 17$, b) $11 \times 17+1.870$ es múltiplo de 187 , c) $11 \times 17+16$ es múltiplo de 187.

Problema 8: si fuera posible, escribe un número que tenga: a) exactamente cuatro divisores naturales, b) más de quince divisores naturales. Si te resultó posible, explica la estrategia que usaste para encontrarlos y si no, explica por qué no es posible. En cualquier caso, fundamenta tu respuesta.

Problema 9: en una estación de colectivos, un bus para con una frecuencia de 18 minutos y el otro lo hace cada 15 minutos, ¿habrá un encuentro posterior después de una coincidencia? Si la respuesta fuera afirmativa, ¿dentro de cuántos minutos, como mínimo, se encontrarán en esa estación, después de haber coincidido en esa estación los dos colectivos? Fundamenta tu respuesta.

Problema 10: se tienen dos cuerdas que miden $240 \mathrm{~cm}$. y $308 \mathrm{~cm}$., se las quiere cortar en trozos que tengan la misma longitud, ¿cuál será la mayor longitud en que se las puede cortar, de forma tal que la longitud de corte sea la misma en ambas cuerdas y que no sobre cuerda? Fundamenta tu respuesta.

Como parte del análisis didáctico, se resolvieron las situaciones problemáticas del instrumento narrando los procesos cognitivos que, a priori, se piensa que elaborarían los futuros profesores, y que serían adecuadas o pertinentes de acuerdo con el nivel educativo para el que está destinado. Por cada práctica de resolución del instrumento se explicitaron los 
elementos de significados primarios y se determinaron las relaciones entre ellos, definidas en términos de funciones semióticas. Más precisamente, por cada práctica se definió una sucesión de funciones semióticas, considerando que generalmente el establecimiento de una de ellas implica la determinación de las anteriores.

A modo de ejemplo se expone el análisis a priori realizado para la situación problemática 7 , donde se explicitan los objetos matemáticos primarios involucrados en la práctica institucional de resolución. En esta práctica no se trata de desplegar todos los conocimientos que dispone el futuro maestro o profesor, sino, más bien, de ilustrar los procesos cognitivos que a priori se piensa que podrían desarrollar, y cuya práctica es considerada adecuada o pertinente.

Se resume a continuación los aspectos centrales señalados para la situación problemática.

a) Se puede afirmar que 17 es divisor de $11 \times 17$, pues es un factor de la descomposición factorial prima de $11 \times 17$. Esto es así, pues teniendo en cuenta la definición de divisor (concepto), podemos decir que 17 es divisor de $11 \times 17$, en tanto existe un número, el 11, que multiplicado por 17 da por resultado $11 \times 17$.

b) El número $11 \times 17+1870$ está escrito teniendo en cuenta la propiedad distributiva. Como $11 \times 17=187$ y $1870=187 \times 10$, y en función de la propiedad distributiva del producto respecto de la suma de números, al número en cuestión $(11 \times 17+$ 1870) se lo puede escribir así: $187+187 \times 10$. Ahora bien, $187+187 \times 10=$ $187 \times(1+10)=187 \times 11$ (concepto y procedimiento), y esta expresión dice que el número dado en la consigna del problema $11 \times 17+1870$ (que también puede escribirse como $187+187 \times 10$ ) es múltiplo de 187 . Esto es así pues 187 es divisor del mismo, por lo que el número propuesto se puede escribir como $187 \times 11$ (concepto y argumento).

Entonces, si un número puede descomponerse en la suma de $n$ productos con un factor común (basándonos en la propiedad distributiva), este número es múltiplo del factor común (propiedad).

c) El número $11 \times 17+16$ está expresado con base en el algoritmo de la división. El dividendo es $203=11 \times 17+16$, el divisor es 17 , el cociente es 11 y el resto 16 .

El primer múltiplo positivo de 187 es el mismo 187 y el múltiplo consecutivo es: $187+187=374$ (procedimiento). El número $11 \times 17+16=203$ está comprendido entre estos dos múltiplos consecutivos de 187 , pues $187<203<374$. Por esta razón se puede afirmar que $11 \times 17+16$ no es múltiplo de 187 , dado que entre dos múltiplos 
consecutivos de un número no existe otro múltiplo (argumento).

La configuración epistémica de la situación problemática 7 resulta ser la que muestra la Figura 2.

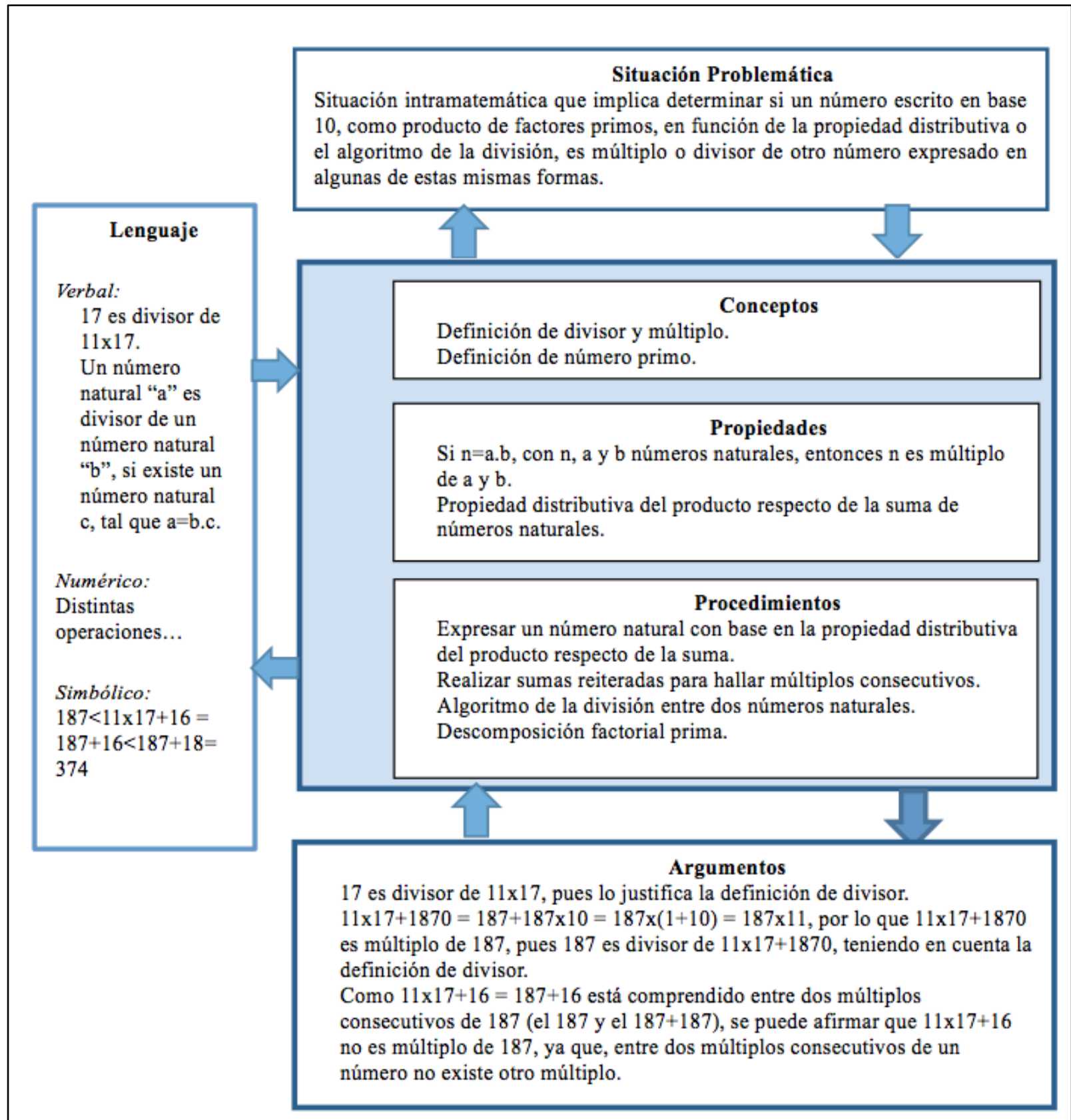

Figura 2 - Configuración Epistémica de la situación problemática 7

Fuente: Elaboración propia

El análisis a priori de la situación problemática y el uso de la herramienta configuración epistémica resultaron útiles para analizar y evidenciar la trama de relaciones que caracteriza la comprensión matemática. En este caso, las funciones semióticas (FS) involucradas en esta configuración son las siguientes:

FS1: entre el problema y el procedimiento de observar si un número p es un número 
primo de la descomposición factorial de un número $n$, para decidir que $\mathrm{p}$ es divisor de $\mathrm{n}$.

FS2: entre el procedimiento que consiste en observar si un número p es un primo de la descomposición factorial de un número $\mathrm{n}$ para decidir que es su divisor y el concepto dado por la definición de divisor.

FS3: entre el problema y el procedimiento que consiste en expresar un número con base en la propiedad distributiva (dejando explícito el factor común) para decidir que el número en cuestión es múltiplo de ese factor común.

FS4: entre el procedimiento que conlleva escribir un número con base en la propiedad distributiva (dejando explícito el factor común) y el concepto de divisor.

FS5: entre el procedimiento de escribir un número con base en la propiedad distributiva (dejando a la vista el factor común) y la propiedad que expresa que cuando un número puede descomponerse en la suma de $\mathrm{n}$ productos con un factor común (propiedad distributiva), entonces este número es múltiplo de ese factor común.

FS6: entre el problema y el procedimiento de acotar el número $11 \times 17+16$ entre dos múltiplos consecutivos de 187.

FS7: entre el procedimiento de acotar el número $11 \times 17+16$ entre dos múltiplos consecutivos de 187, y el argumento que explica que aquel número no es múltiplo de 187, ya que entre dos múltiplos consecutivos de un número no existe otro múltiplo.

Cabe aclarar que tanto en estas FS, enmarcadas en la referencia institucional, como en aquellas identificadas en las prácticas personales de los futuros profesores, que se exponen más adelante, la expresión corresponde al primer objeto matemático señalado en cada una de ellas, mientras que el contenido, al segundo objeto, el que queda explícito luego de la conjunción $y$.

A modo de complemento del análisis expuesto recientemente, pueden apreciarse otras prácticas institucionales de referencia en el Cuadro 1, el cual contiene la matriz de desempeños o rúbrica asociada a la situación problemática 7. Se decide exponer dichas prácticas en el formato mencionado para facilitar la comprensión de un lector que no es experto en el marco teórico de este trabajo, a pesar de que en el mismo se pueden distinguir los objetos matemáticos primarios.

\begin{tabular}{|c|c|c|c|}
\hline \multicolumn{4}{|c|}{ Diferentes desempeños para la situación-problema 7} \\
\hline Novato & Aprendiz & Experto & Distinguido \\
\hline Usar definiciones & (a) Dividir 187 entre & (a) 17 es divisor de 187, pues & a) 17 es divisor de $11 \times 17$, \\
\hline rudimentarias de & 17. (procedimiento) & cumple con la definición de & porque es un factor de la \\
\hline Divisor $\quad \mathrm{y}$ & Como se obtiene & divisor, ya que existe el 11 , tal & descomposición factorial \\
\hline Múltiplo & cociente 11 y resto 0 & que $11 \times 17=187$ (concepto $y$ & prima de $11 \times 17$ (concepto $\mathrm{y}$ \\
\hline proceder así: & se tiene que 17 es & procedimiento). & argumento). En efecto, todo \\
\hline a) 17 es divisor de & divisor de 187 & (b) El número $11 \times 17+1.870$ & factor $p$ de la descomposición \\
\hline
\end{tabular}




\begin{tabular}{|c|c|c|c|}
\hline 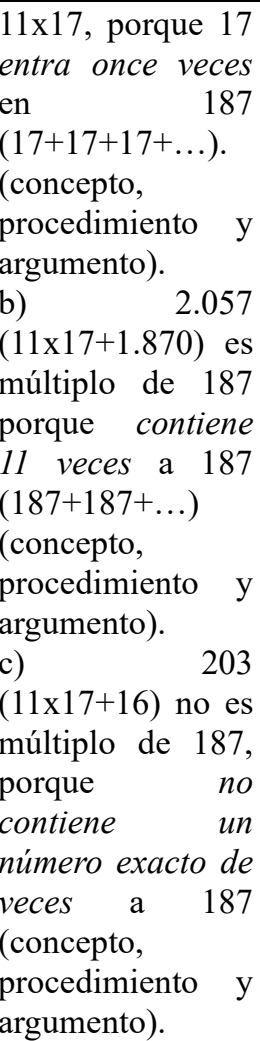 & 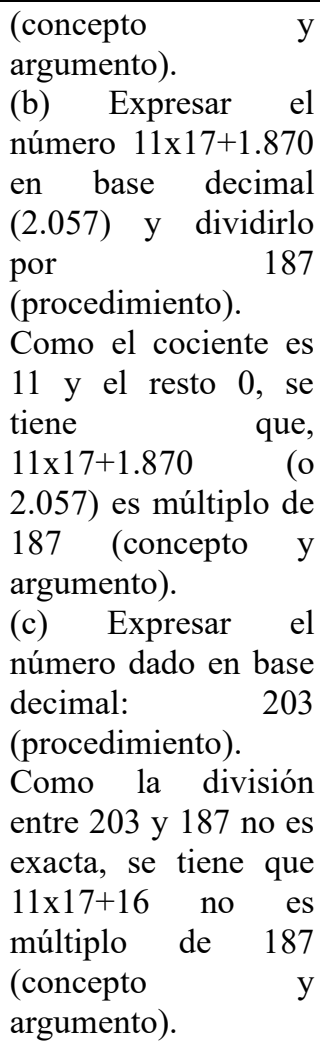 & $\begin{array}{l}\text { puede escribirse como: } \\
187+187 \times 10 \text {, que es igual a } \\
187 \times(10+1) \text { = } 187 \times 11 \\
\text { (procedimiento), o sea que } \\
11 \times 17+1870 \text { es múltiplo de } \\
187 \text {, pues este número por } \\
\text { definición es divisor de } \\
11 \times 17+1870 \text { (concepto). } \\
\text { (c) El número } 11 \times 17=187 \text { es } \\
\text { el primer múltiplo de } 187 \text {, } \\
\text { mientras que el siguiente } \\
\text { múltiplo es } 187+187 \\
\text { (concepto). Como } 11 \times 17+16 \\
=187+16 \text { está comprendido } \\
\text { entre los dos múltiplos } \\
\text { consecutivos de } 187 \text { recién } \\
\text { indicados, 11x17+16 no es } \\
\text { múltiplo de } 187 \text { dado que, } \\
\text { entre dos múltiplos } \\
\text { consecutivos de un número, } \\
\text { no existe otro múltiplo } \\
\text { (propiedad y argumento). }\end{array}$ & $\begin{array}{l}\text { factorial prima de un número a } \\
\text { es su divisor, pues a puede } \\
\text { expresarse como el producto } \\
\text { entre p y q, siendo q el } \\
\text { producto de todos los otros } \\
\text { factores primos de la } \\
\text { descomposición factorial } \\
\text { prima de a, cumpliendo } \\
\text { entonces con la definición de } \\
\text { divisor (concepto } \\
\text { argumento). } \\
\text { b) } 11 \times 17+1.870 \text { es múltiplo de } \\
187 \text {, ya que dicho número es } \\
\text { la suma de otros dos múltiplos } \\
\text { de } 187 \text {, } 11 \times 17 \text { y } 1.870 \\
\text { (concepto y propiedad). } \\
\text { c) } 11 \times 17+16 \text { no es múltiplo de } \\
187 \text {, porque de los dos } \\
\text { sumandos, uno solo de ellos, } \\
\text { el } 11 \times 17 \text {, es múltiplo de } 187 \\
\text { (concepto y propiedad). }\end{array}$ \\
\hline
\end{tabular}

Cuadro 1 - Rúbrica de desempeños de la situación problemática 7 Fuente: Elaboración propia

Para valorar la comprensión alcanzada por futuros maestros y profesores de matemática, es necesario determinar niveles de comprensión. En cada nivel, se explicitan las funciones semióticas pertenecientes al mismo y se procede al análisis de las redes de relaciones conceptuales involucradas. En el análisis, se pone énfasis fundamentalmente en el contenido de las funciones semióticas. Estos niveles se pueden definir en términos de la variedad, riqueza matemática y complejidad de las relaciones conceptuales involucradas en las funciones semióticas pertenecientes a ellos.

Además de la clasificación en niveles de las funciones semióticas, el análisis de redes de relaciones conceptuales puestas en funcionamiento por los futuros maestros y profesores de matemática aporta sustanciales elementos para valorar la comprensión. Estas funciones semióticas pueden clasificarse:

Función semiótica actuativa: cuando su contenido es un procedimiento, una técnica, una manera de hacer.

Función semiótica argumentativa: cuando su contenido es un argumento. Se clasifican, a su vez:

- Argumentativa conceptual: la que tiene como contenido una justificación dada a través de una definición o concepto. 
- Argumentativa proposicional: cuando su contenido es una justificación basada en una propiedad.

- Argumentativa con contraejemplo: cuando su contenido es una justificación dada a partir de un contraejemplo.

- Argumentativa deductiva formal: cuando su contenido es una justificación basada en una deducción escrita formalmente en base a una cadena de implicaciones, equivalencias o igualdades.

Una distinción en niveles de comprensión de las prácticas de estudiantes (futuros profesores) que se analizan, resulta ser el siguiente: al primer nivel pertenecen las funciones semióticas actuativas (sólo se emplean procedimientos, técnicas, rutinas y algoritmos); al segundo, las funciones semióticas argumentativas conceptuales, proposicionales y con contraejemplos; mientras que el tercer nivel incluye las funciones semióticas argumentativas deductivas formales.

A modo de ejemplo, se describe la red de relaciones conceptuales involucradas en las funciones semióticas argumentativas deductivas formales, identificadas en las prácticas personales que se analizan (máximo nivel de comprensión que podrían evidenciar los futuros maestros y profesores de matemática).

FS que relaciona el problema de determinar si un número expresado con base en la propiedad distributiva es múltiplo de otro, y la deducción: $11 \times 17+1180=187+187 \times 10=$ $187 x(1+10)=187 x 11$, por lo tanto, $11 x 17+1180$ es múltiplo de 187.

Hay un reconocimiento que $11 \times 17+1180$ es un número. No hay necesidad de obtener la expresión en base decimal del mismo para responder la pregunta acerca de si es múltiplo de 187. En el desarrollo de la deducción se ponen en funcionamiento conocimientos de tipo procedimental, como sumar y obtener descomposiciones multiplicativas de un número. Asimismo, se emplean definiciones y propiedades: la definición de múltiplo (la que indica que un número es múltiplo de un factor de una descomposición multiplicativa del mismo) y la propiedad distributiva del producto respecto de la suma de números naturales.

FS que relaciona el problema de determinar el orden que se establece entre dos números, cuando uno es divisor de otro, y la deducción: si a y b son números naturales y a $\mid b$, se sabe que $a \leq b$. Si $a \mid b$, entonces, $a \mid-b$, siendo $a>-b$, considerando que a y $b$ son números naturales. En este caso, al ser el divisor mayor que el número, el orden que se establece entre a y b en $Z$ no es el mismo que en $N$.

Esta argumentación deductiva, desarrollada en lenguaje simbólico y natural, permite responder la pregunta planteada. Puede apreciarse el establecimiento de una relación entre las 
nociones de divisor y divide, ya que en el problema se pide relacionar dos números, cuando uno de ellos es divisor del otro, y en la argumentación aparece planteada la relación divide, inclusive, con la notación pertinente. En el desarrollo de la deducción se aprecia la correcta identificación de los números a y b en la relación a $\mid \mathrm{b}$, como así también, el uso de la propiedad: si a $\mid \mathrm{b}$, entonces, $\mathrm{a} \mid-\mathrm{b}$. La misma permite determinar que la relación entre a y $\mathrm{b}$, cuando el primero es divisor del segundo, no es la misma en $\mathrm{N}$ y Z.

FS que relaciona un problema de mínimo común múltiplo, y la deducción: como hay una relación de 5/6 entre las demoras de ambos buses, el que tarda más tiempo (18 min.) dará 5 vueltas y el que tarda menos (15 min.), 6 vueltas, para volver a encontrarse. El primero realiza 5 vueltas en 90 min. y el otro, 6 vueltas en 90 min. O sea, se volverán a encontrar a los $90 \mathrm{~min}$.

Esta deducción, desarrollada en lenguaje natural, permite responder la cuestión planteada. Se establece una relación entre los tiempos de demora de los buses a través de una razón aritmética. Se deduce, a partir de esta relación, la cantidad de vueltas que deben dar ambos buses antes de volver a encontrase. Hay un reconocimiento del hecho de que el bus que tarda más tiempo en regresar a la estación realizará menos vueltas hasta volver a encontrarse con el otro bus que tarda menos, y viceversa. Con estos conocimientos, multiplicando números naturales, se puede resolver el problema planteado. A su vez, este tipo de prácticas permite abordar problemas de mínimo común múltiplo entre dos números naturales.

\section{Reflexiones finales}

El diseño, la descripción y el análisis - previo y posterior - presentados, sobre el instrumento de indagación para valorar la comprensión alcanzada en Divisibilidad, permiten revisar las prácticas que llevan a cabo los futuros profesores de matemática y, al mismo tiempo, explorar y potenciar la faceta del conocimiento epistémico sobre esta temática en los formadores de profesores. Para estos últimos, el análisis didáctico de tareas también ayuda a contar con buenas herramientas para interpretar las prácticas matemáticas de los estudiantes, y mejorar las condiciones para elaborar y diseñar propuestas de enseñanza pertinentes.

Asimismo, el análisis de las prácticas de los futuros maestros o profesores de matemática, en términos de funciones semióticas, pone en evidencia un gran número de objetos matemáticos primarios pertenecientes a la Divisibilidad. Esta situación activa un conglomerado de funciones semióticas entre los objetos primarios que es diferente para cada uno de los niveles de comprensión que puedan definirse localmente. En este sentido, las 
funciones semióticas tienen un papel importante en el proceso relacional entre entidades (o grupos de ellas) y permiten entender la comprensión que alcanzan los sujetos sobre un objeto matemático (FONT, 2002).

En las prácticas de futuros profesores de matemática se pueden establecer, claramente, tres niveles de comprensión. Estos se determinan en términos de funciones semióticas que el mismo sujeto puede establecer, en unas circunstancias fijadas, en las que se pone en juego al objeto como funtivo (expresión o contenido). Así, los que sólo establecen funciones semióticas actuativas se los puede ubicar en el primer nivel de comprensión alcanzado sobre objetos matemáticos de la Divisibilidad. Aquellos que, además, pueden establecer funciones semióticas argumentativas (conceptuales, proposicionales y a partir de contraejemplos), se encuentran en el segundo nivel de comprensión. Finalmente, los que pueden establecer funciones semióticas argumentativas deductivas formales, se ubicarían en un tercer nivel de comprensión sobre Divisibilidad.

\section{Referencias}

ARENDS, R. Learning to teach. 6. ed. Boston: Mc GrawHill, 2004.

BODÍ, S. Análisis de la comprensión de divisibilidad en el conjunto de los Números Naturales. 2006. Tesis (Doctorado en Educación) - Facultad de Educación, Universidad de Alicante, Alicante, 2006.

BODÍ, S.; VALLS, J.; LLINARES, S. La comprensión de la divisibilidad en N. Un análisis implicativo. En: GRAS, R.; ORÚS, B.; PINAUD, B. (Ed.). Nouveaux Apports Théoriques à l'Analyse Statistique Implicative et Applications. Castellón: Université Jaume I, 2007. p. 99-110.

BROWN, A. Patterns of thought and prime factorization. In: CAMPBELL, S. R.; ZAZKIS, R. (Ed.). Learning and teaching number theory: Research in cognition and instruction. Westport: Ablex Publishing, 2002. p. 131-137.

COBB, P.; GRAVEMEIJER, K. Experimenting to support and understand learning processes. In: KELLY, A.; LESH, R.; BAEK, J. (Ed.). Handbook of design research methods in education: Innovations in science, technology, engineering and mathematics learning and teaching. New Jersey: Lawrence Erlbaum Associates, 2008. p. 68-95.

CONFERENCE BOARD OF THE MATHEMATICAL SCIENCES (CBMS). The mathematical education of teachers. Providence: American Mathematical Society, 2001.

D’AMORE, B.; FONT, V.; GODINO, J. D. La dimensión metadidáctica en los procesos de enseñanza y aprendizaje de la matemática. Paradìgma, Maracay, v. 28, n. 2, p. 49-77, dic. 2007.

DISTÉFANO, M. L. Procesos de significación para algunos símbolos matemáticos en estudiantes universitarios. 2017. Tesis (Doctorado en Enseñanza de las Ciencias mención Matemática) - Facultad de Ciencias Exactas, Universidad Nacional del Centro de la Provincia de Buenos Aires, Argentina. 2017. 
DUBINSKY, E. Reflective abstraction in advanced mathematical thinking. In: TALL, D (Ed.).

Advanced Mathematical Thinking. Boston: Kluwer Academic Publishers, 1991. p. 95-123.

ESPINOZA, R. Estudios didáctico-matemáticos de prácticas asociadas a la Divisibilidad en Números Enteros. 2012. 115 f. Disertación (Maestría en Docencia Universitaria) - Facultad de Humanidades, Universidad Nacional del Nordeste, Corrientes, 2013.

ESPINOZA, R. La comprensión alcanzada por estudiantes de Profesorado en Matemática, referida a la Divisibilidad, al comenzar la Universidad. 2018. $282 \mathrm{f}$. Tesis (Doctorado en Ciencias Humanas y Sociales) - Facultad de Humanidades y Ciencias Sociales, Universidad Nacional de Misiones, Posadas, 2019.

ETCHEGARAY, S. Análisis epistemológico y didáctico de nociones elementales de Teoría de Números. 1998. Disertación (Maestría en Didáctica de las Matemáticas) - Facultad de Ciencias Exactas, Físico-Químicas y Naturales, Universidad Nacional de Río Cuarto, Río Cuarto, 1998.

FELDMAN, Z. Describing Pre-Service Teachers' Developing Understanding of Elementary Number Theory Topics. 2012. Tesis (Doctorate in Education) - School of Education, Boston University, Boston, 2012.

FONT, V. Una organización de los programas de investigación en Didáctica de las Matemáticas. EMA, Bogotá, v. 7, n. 2, p. 127-170, jul. 2002.

FONT, V. Las funciones y la competencia disciplinar en la formación docente matemática. UNORevista de Didáctica de las Matemáticas, Barcelona, v. 56, n. 1, p. 86-94, 2011.

GODINO, J. D. Significado y comprensión en matemáticas. UNO - Revista de Didáctica de las Matemáticas, Barcelona, v. 25, n. 0, p. 77-7, jul. 2000.

GODINO, J. D. Teoría de las funciones semióticas: Un enfoque ontológico semiótico de la cognición e instrucción matemática. Granada: Departamento de Didáctica de la Matemática de la Universidad de Granada, 2003. Disponible en: https://www.ugr.es/ jgodino/funciones-semioticas/monografiatfs.pdf. Acceso: 3 may. 2019.

GODINO, J. D. Indicadores de la idoneidad didáctica de procesos de enseñanza y aprendizaje de las matemáticas. Cuadernos de Investigación y Formación en Educación Matemática, San José, v. 11, n. 3, p. 111-132, jun. 2013.

GODINO, J.; BATANERO, C.; FONT, V. The onto-semiotic approach to research in mathematics education. ZDM, Hamburg, v. 39, n. 1-2, p. 127-135, ene. 2007.

INSTITUTO NACIONAL DE FORMACIÓN DOCENTE (INFD). Proyecto de mejora para la formación inicial de profesores para el nivel secundario. Área: Matemática. Ministerio de Educación, Instituto Nacional de Formación Docente y Secretaría de Políticas Universitarias. Buenos Aires, 2010. Disponible en: https://cedoc.infd.edu.ar/upload/Matematica.pdf. Acceso: 03 may. 2019.

LÓPEZ, A. Significados de la relación de divisibilidad de maestros en formación manifestados en el desarrollo de un modelo de enseñanza. 2015. Tesis (Doctorado en Ciencias de la Educación) Facultad de Ciencias de la Educación, Universidad de Granada, Granada, 2016.

NATIONAL COUNCIL OF TEACHING MATHEMATICS (NCTM). Curriculum and evaluation standards for school mathematics. Reston: The Council, VA 20191, EE. UU., 1989.

PINO-FAN, L.; GODINO, J.; FONT, V. Faceta epistémica del conocimiento didáctico-matemático sobre la derivada. Educação Matemática Pesquisa, São Paulo, v. 13, n. 1, p. 141-178, mar. 2011. 
PINO-FAN, L.; GODINO, J.; FONT, V. Diseño y aplicación de un instrumento para explorar la faceta epistémica del conocimiento didáctico-matemático del futuro profesor sobre la derivada (Segunda parte). REVEMAT: Revista Eletrônica de Educação Matemática, Florianópolis, v. 8, n. edição especial, p. 1-47, dic. 2013.

VALLEJOS VARGAS, E. Análisis y propuesta en torno a las justificaciones en la enseñanza de la divisibilidad en el primer grado de secundaria. Lima: Pontificia Universidad Católica del Perú, 2012.

VERGNAUD, G. Multiplicative conceptual field: what and why? In: GUERSON, H.; CONFREY, J. (Ed.). The development of multiplicative reasoning in the learning of mathematics. Albany: SUNY, 1994. p. 41-59.

ZAZKIS, R. Factors, divisors and multiples: Exploring the web of students' connections. In: DUBINSKY, E.; SCHOENFELD, A.; KAPUT, J. (Ed.). Research in Collegiate Mathematics Education IV. Providence: CBMS, 2000. p. 210-238.

ZAZKIS, R. Múltiplos, divisores y factores: explorando la red de conexiones de los estudiantes. RELIME - Revista Latinoamericana de Investigación en Matemática Educativa, Ciudad de México, v. 4, n. 1, p. 63-92, mar. 2001.

ZAZKIS, R.; CAMPBELL, S. Divisibility and Multiplicative structure of natural numbers: preservice teacher's understanding. Journal for Research in Mathematics Education, Newark, v. 27, n. 5, p. 540-563, nov. 1996.

ZAZKIS, R.; GADOWSKY, K. Attending to transparent features of opaque representations of natural numbers. In: CUOCO, A. (Ed.). NCTM 2001 Yearbook: The roles of representation in school mathematics. Reston: NCTM, 2001. p. 41-52.

ZAZKIS, R.; LILJEDAH, P. Understanding primes: the role of representation. Journal for Research in Mathematics Education, Newark, v. 35, n. 3, p. 164-186, may. 2004.

Submetido em 23 de Maio de 2019 Aprovado em 09 de Dezembro de 2019. 